Montana Tech Library

Digital Commons @ Montana Tech

Fall 10-15-2016

Understanding risk factor patterns in ATV fatalities: A recursive partitioning approach

John Rosecrance

David P. Gilkey

Elise Lagerstrom

Sheryl Magzamen

Lorann Stallones

Follow this and additional works at: https://digitalcommons.mtech.edu/shih

Part of the Occupational Health and Industrial Hygiene Commons 


\title{
Understanding risk factor patterns in ATV fatalities: A recursive partitioning approach
}

\author{
Elise Lagerstrom, ${ }^{\mathrm{a}, *}$ Sheryl Magzamen, ${ }^{\mathrm{a}, \mathrm{c}}$ Lorann Stallones, ${ }^{\mathrm{b}, \mathrm{c}}$ David Gilkey, ${ }^{\mathrm{a}}$ John Rosecrance ${ }^{\mathrm{a}, \mathrm{c}}$ \\ a Department of Environmental and Occupational Health Sciences, Colorado State University, Fort Collins, CO, United States \\ b Department of Psychology, Colorado State University, Fort Collins, CO, United States \\ c Colorado School of Public Health, Colorado State University, Fort Collins, CO, United States
}

\section{A R T I C L E I N F O}

Article history:

Received 19 May 2016

Received in revised form 23 August 2016

Accepted 5 October 2016

Available online 15 October 2016

\section{Keywords:}

ATV

All-terrain vehicle

Risk factors

Epidemiologic triangle

Helmet use

\begin{abstract}
A B S T R A C T
Introduction: Although there are hundreds of ATV-related deaths each year in the United States, contributing factors have not been clearly identified. The purpose of this study was to investigate associations between factors contributing to ATV fatalities using the agent-host-environment epidemiological triangle. Method: Incident reports of ATV fatalities occurring between 2011 and 2013 were obtained from the United States Consumer Product Safety Commission (CPSC). Narrative reports included details of the decedent and a description of the ATV crash. A chi-square automatic interaction detector (CHAID) analysis was performed for three major risk factors representing each facet of the epidemiologic triangle: helmet use (host), type of crash (agent), and location where death occurred (environment). The output of the CHAID analysis is a classification tree that models the relationship between the predictor variables and a single outcome variable. Results: A total of 1193 ATV fatalities were reported to the CPSC during the 3-year study period. In cases with known helmet and/or drug and alcohol use status, descriptive statistics indicated helmets were not worn in $88 \%$ of fatalities and use of alcohol or drugs was present in $84 \%$ of fatalities. Reoccurring factors within the CHAID analysis included age, helmet use, geographic region of the country, and location (e.g., farm, street, home, etc.) at the time of death. Within the three CHAID models, there were seven significant partitions related to host, one related to agent, and eight related to the environment. Conclusions: This research provides a model for understanding the relationship between risk factors and fatalities. The combination of the CHAID analysis method and the epidemiologic triangle allows for visualization of the interaction between host-agent-environment factors and fatalities. Practical applications: By modeling and characterizing risk factors associated with ATV fatalities, future work can focus on developing solutions targeted to specific factions of ATV users.
\end{abstract}

(c) 2016 National Safety Council and Elsevier Ltd. All rights reserved.

\section{Introduction}

All-terrain vehicles (ATVs) were marketed in the United States in the 1970s as an occupational tool that bridged the gap between the tractor and horse (Balthrop, Nyland, \& Roberts, 2009). While ATVs are still used for occupational purposes, there has been a substantial amount of growth in the number of recreational riders in the United States. In 1990, there were an estimated 1.8 million ATVs in use in the United States (Topping \& Garland, 2014); in 2011, this number rose to 10.7 million (Topping \& Garland, 2014). According to the United States Consumer Product Safety Commission (CPSC), the estimated number of four-wheeled ATV-related injuries in 1990 was 30,800 and the estimated number of deaths was 152 (Topping \& Garland, 2014). In

\footnotetext{
* Corresponding author at: Colorado State University, 1681 Campus Delivery, Fort Collins, CO 80523-1681, United States.

E-mail address: lagerstrome@gmail.com (E. Lagerstrom).
}

2011, these numbers rose to 105,000 injuries and 666 deaths (Topping \& Garland, 2014), a $241 \%$ and $338 \%$ increase, respectively.

Injury prevention strategies focused on the reduction in ATV-related injuries and fatalities must address the various risk factors associated with these incidents. The epidemiologic triangle has been a useful model to better understand the complexity of risk factors contributing to injuries and fatalities (Hulme \& Finch, 2015; Runyan, 2003). Risk factors for ATV fatalities can be categorized to fit the three elements of the Epidemiologic Triangle: host, agent, and environment.

Host factors relate to the demographics or behaviors of the ATV operator such as gender (Breslau, Stranges, Gladden, \& Wong, 2009; Goldcamp, Myers, Hendricks, Layne, \& Helmkamp, 2006; Helmkamp, Aitken, \& Lawrence, 2009; O'Connor, Hanks, \& Steinhardt, 2009; Rechnitzer, Grzebieta, McIntosh, \& Simmons, 2013; Rodgers, 2008; Rodgers \& Adler, 2001), age (Balthrop et al., 2009; Helmkamp \& Carter, 2009; Helmkamp, Marsh, \& Aitken, 2011; O'Connor et al., 2009; Rodgers, 2008; Rodgers \& Adler, 2001), experience and training 
(Goldcamp et al., 2006; O'Connor et al., 2009; Rodgers \& Adler, 2001), use of personal protective equipment(Fleming, 2010; Mangus, Simons, Jacobson, Streib, \& Gomez, 2004; Myers, Cole, \& Mazur, 2009), risk tolerance and perception of the operator (Fleming, 2010), and use of drugs and alcohol (Fleming, 2010). Agent factors are those that relate to the energy of the ATV. This includes speed capabilities (Helmkamp et al., 2011), the stability and weight of the vehicle (Percy \& Duffey, 1989), and mechanical failures (Fleming, 2010). Environmental factors include both the physical and social environment. While many of the environmental risk factors associated with ATV fatalities are controllable, such as the road surface (off-road vs. asphalt), others, such as weather, are not easily controlled. Factors related to the social environment include behaviors associated with group riding and ATV operation with passengers (Fleming, 2010).

Using the epidemiologic triangle as a classification method for understanding underlying risk factors for fatalities may lead to safety and protection innovations. Identification of the predominant risk factor classification, host, agent, or environment, will help direct resources to the appropriate intervention strategy, whether that be legislation, engineering controls, advances in the effectiveness or use of personal protective equipment, or operator training. The Haddon Matrix is a method of examining possible interventions to the different aspects of the epidemiologic triangle at different points in the accident timeline (Haddon, 1968). Haddon stated, "There are essentially three major portions or phases of the sequence of events leading up to the end results, during which causal factors are active and countermeasures can be undertaken" (Haddon, 1968). Haddon originally called these phases "the phases of social concern." In most applications, these phases would later be simplified to correspond to the pre-accident, accident, and post-accident phase of the injury event (Haddon, 1968). Application of the Haddon Matrix allows for evaluation of the hazards of the accident, as well as identification of a set of solutions that could be applied to risks associated with that injury event.

To date, the preponderance of intervention strategies designed to reduce the high injury rate associated with ATVs have targeted host and agent risk factors rather than environmental factors. In 1988, the CPSC proposed a 10 -year plan to increase ATV safety by prohibiting the sale of three-wheeled ATVs, establishing age recommendations for ATV operators, and implementing training programs for new ATV purchasers (Rodgers \& Adler, 2001). This plan was successful in reducing ATV-related fatalities. In 1988, the estimated rate of ATV-related fatalities in the United States was 179 per million ATVs in use (Topping \& Garland, 2014). By 1998, the estimated ATV fatality rate had declined to 81 per million ATVs in use (Topping \& Garland, 2014). However, just one year after the plan's expiration, in 1999, the fatality rate rose to 105 per million ATVs in use (Topping \& Garland, 2014).

Due to the success of the CPSC's initial safety campaign, and the increasing injury and fatality rates after its expiration (Balthrop et al., 2009), the CPSC enacted the 2008 Consumer Product Safety Improvement Act (CPSIA). This act was designed to increase the safety of the ATV and reduce the fatality rate by altering design, promoting awareness of safe ATV operation through training and marketing, and targeting a high-risk age demographic of users, children and youth.

Similar to the 1988 plan, the 2008 CPSIA was designed to combat the increasing ATV fatality rate from the host and agent aspects of the epidemiologic triangle. The 2008 CPSIA's campaign intended to reduce the risk to children and youth by recommending smaller engine sizes and a maximum speed based on the child's age (Catenacci, 2009; Fleming, 2010; Goldcamp et al., 2006). Agent-related risk factors were further controlled by extending the ban on the sale of three wheeled ATVs. While this mandatory design change from three to four wheeled vehicles increased the stability and balance of the ATV (Percy \& Duffey, 1989), a literature review (Balthrop et al., 2009) suggested that factors related to the social environment, consumer culture and demand, drove the industry to increase the speed and power of ATVs. The increase in ATV power may have offset the benefits of improved design and resulted in increased severity of ATV injuries (Balthrop et al., 2009). The interaction between agent and environmental factors demonstrates the complexity of developing and implementing intervention strategies for improving ATV safety.

The purpose of this study was to examine and model contributing factors of ATV injuries and fatalities using three years of CPSC fatality reports. The Epidemiological Triangle was used to model the complexity of factors associated with ATV-related fatalities. Use of the epidemiologic triangle allows for an improved understanding of the different sources of risk and the interaction between the operator, machine and environmental conditions.

\section{Methods}

\subsection{Data source}

ATV incident data were obtained from CPSC In-Depth Investigation Files (INDP) for the years 2011-2013. The INDP files are reports of incidents and fatalities that have been investigated by the CPSC based on interviews or on-scene investigations. Each INDP report contains demographic information as well as a narrative report of the incident. The CPSC identifies incidents for investigation from a variety of different sources, the main sources being: news reports, death certificate files (DTHS), and the Medical Examiners and Coroners Alert Project (MECAP). Once identified, these incidents are investigated by CPSC personnel either by phone, onsite, or using other methods.

\subsection{Data collection}

Each INDP report was read and manually coded into a Microsoft Excel File. The internal reliability of the coding was checked using two different coders. One coder entered the data from all fatality reports while a second coder entered data from a randomly selected sample of $10 \%$ of reports. The reliability between the two coders was analyzed for each variable using Cohen's Kappa. Kappa values of 0.81 or higher were considered to have excellent agreement (Landis \& Koch, 1977).

To apply the epidemiological triangle to each case, variables from the demographic and narrative sections of the INDP reports were categorized as pertaining either to the agent, the host, or the environment. Variables pertaining to the host (ATV user) involved in the crash included: age, sex, status as the driver or passenger of the ATV, helmet use (yes, no, unknown), and use of alcohol or drugs (yes, no, unknown). Variables pertaining to the agent (the energy of the ATV) involved in the crash included: type of crash (overturn, ejection, collision, or other). Variables pertaining to the environment (physical or social) involved in the crash included: date, season, region, location of crash, number of users on ATV, and if another vehicle was involved.

\subsection{Data analysis}

Descriptive statistics were calculated using SAS version 9.3. Frequency statistics were determined for categorical variables and univariate analyses were performed on continuous variables. Chi-square tests for equal proportions were performed for the following variables: sex, diagnosis, body part injured, season, region, and location type.

A chi-square automatic interaction detector (CHAID) analysis was conducted using JMP Pro 11. This analysis tool was used to identify characteristics associated with different outcomes or variables. Like a traditional regression, a CHAID analysis sets one variable as the dependent characteristic (outcome measure) with all other variables as independent predictors. The outcome of the CHAID analysis is a classification tree that illustrates the hierarchical relationship among the outcome measure and independent predictor variables. The advantage of CHAID over regression is the ability to illustrate the clustering of variables. The method employs an iterative process, by first examining the cross tabulation between each of the predictor variables and the 
outcome and tests for significance using a chi-square tests for independence test. CHAID will select the predictor that is most significant (smallest $p$ value), then iterate through the remaining predictor variables to select the covariates to partition the data (SPSS Inc., 2002). The partitioning of categorical data was performed based on the $\mathrm{G}^{2}$ statistic and calculated LogWorth where LogWorth = $\log (p$-value $)($ SAS Institute Inc., 2014b).

CHAID was used to create three different classification trees based on the following outcome measures: helmet use (yes, no, unknown), crash type, and location type. These three variables were chosen as outcome measures because they each represent a different facet of the epidemiologic triangle: helmet use (host), type of loss of control event (agent), and location type (environment). Only variables categorized as host, agent, or environment were used as predictor variables. The classification trees had a minimum group size of 25 and cases with an unknown value for the dependent characteristic were removed from the analysis. Based on a recommendation in the literature, trees were split until the LogWorth of the split was less than or equal to two (SAS Institute Inc., 2014a). A LogWorth of two corresponds to a significant p-value of 0.01 .

After creation of the classification trees, each partition was categorized as a host, agent, or environment division. By categorizing the partitions, it is possible to illustrate which classification of variables lead to the outcome measure and if overlap exists between host, agent, and environmental factors.

\section{Results}

\subsection{Descriptive statistics}

A total of 1230 reports for the study period were obtained from CPSC. The 1230 reports contained non-injury, mortality, and morbidity cases. Due to the limited number of narrative reports of non-injury or morbidity cases ( $n=37$ ), this study was limited to mortality cases. After removing cases of non-injury and morbidity, a total of 1193 (97\%) reports remained.

Demographic statistics were calculated from the 1193 cases (Table 1$)$. Of the total fatalities, $85 \%$ were male $\left(\chi^{2}=570.52\right.$, $p<0.0001$ ). Median age at time of death was found to be 36 (range, $1-91)$. Of the sample, $14 \%$ were less than $16(n=167)$ and $12 \%$ were greater than 64 years of age $(n=145)\left(\chi^{2}=880.10, p<.0001\right)$. All kappa values were found to have either substantial or almost perfect agreement between the two coders.

Within the agent and injury varialbes, $60 \%$ of all fatalities were classified as head injuries. Of the cases with known helmet use status, only $12 \%$ of cases were wearing a helmet at the time of death. There is a signifcant opportunity to intervene in the number of head injuries by increasing the number of riders wearing helmets.

Season and regional differences also existed in the number of INDP reports completed during the study period. A chi-square test demonstrated significant differences in the number of reports of fatalities by season, with summer as highest $\left(\chi^{2}=216.71, p<0.0001\right)$ and the South as the region with the highest proportion of fatalities $\left(\chi^{2}=\right.$ $355.95, p<0.0001$ ).

\subsection{CHAID}

The following sections describe the associations between the independent predictor variables and outcome measures. The outcome measures chosen as the basis for the CHAID models were: helmet use, crash type, and location type.

\subsubsection{Helmet use (Fig. 1)}

Among the 70\% of cases where helmet use status was known $(n=$ 810 ), $88 \%$ of ATV users involved in fatal cases were not wearing a helmet at the time of death. The covariate most strongly associated with helmet
Table 1

Descriptive statistics for host-agent-environment variables.

\begin{tabular}{|c|c|c|c|c|}
\hline & & & Percent $(n)$ & $p^{*}$ \\
\hline \multirow[t]{15}{*}{$\begin{array}{l}\text { Host } \\
\text { variables }\end{array}$} & \multirow[t]{2}{*}{ Gender } & Male & $\begin{array}{l}84.58 \% \\
(1009)\end{array}$ & \multirow[t]{2}{*}{$<.0001$} \\
\hline & & Female & $15.42 \%(184)$ & \\
\hline & \multirow[t]{4}{*}{ Age } & $<16$ & $14.00 \%$ (167) & \multirow[t]{4}{*}{$<.0001$} \\
\hline & & $16-64$ & $73.76 \%(880)$ & \\
\hline & & $>64$ & $12.24 \%(145)$ & \\
\hline & & Unknown & (1) & \\
\hline & \multirow[t]{3}{*}{ Driver or passenger } & Driver & $\begin{array}{l}91.12 \% \\
(1078)\end{array}$ & \multirow[t]{3}{*}{$<.0001$} \\
\hline & & Passenger & $8.88 \%(105)$ & \\
\hline & & Unknown & $(10)$ & \\
\hline & \multirow[t]{3}{*}{ Helmet } & Yes & $11.85 \%(96)$ & \multirow[t]{3}{*}{$<.0001$} \\
\hline & & No & $88.15 \%(714)$ & \\
\hline & & Unknown & $(383)$ & \\
\hline & \multirow[t]{3}{*}{ Alcohol or drug use } & Yes & $84.27 \%(241)$ & \multirow[t]{3}{*}{$<.0001$} \\
\hline & & No & $15.73 \%(45)$ & \\
\hline & & Unknown & $(907)$ & \\
\hline \multirow{16}{*}{$\begin{array}{l}\text { Agent/injury } \\
\text { variables }\end{array}$} & \multirow[t]{4}{*}{ Type of crash } & Collision & $42.92 \%(512)$ & \multirow[t]{4}{*}{$<.0001$} \\
\hline & & Overturn & $37.38 \%(446)$ & \\
\hline & & Ejection & $11.74 \%(140)$ & \\
\hline & & Unknown/other & $7.96 \%(95)$ & \\
\hline & \multirow[t]{5}{*}{ Diagnosis } & Internal injuries & $68.15 \%(813)$ & \multirow[t]{5}{*}{$<.0001$} \\
\hline & & Other & $12.99 \%(155)$ & \\
\hline & & Fracture & $6.79 \%(81)$ & \\
\hline & & Anoxia & $6.37 \%(76)$ & \\
\hline & & Crushing & $5.70 \%(68)$ & \\
\hline & \multirow[t]{7}{*}{ Body part injured } & Head & $59.98 \%(655)$ & $<.0001$ \\
\hline & & Multiple & $17.03 \%(186)$ & \\
\hline & & Upper trunk & $16.48 \%(180)$ & \\
\hline & & Neck & $4.67 \%(51)$ & \\
\hline & & Lower trunk & $1.65 \%(18)$ & \\
\hline & & Leg & $0.18 \%(2)$ & \\
\hline & & Unknown & $(101)$ & \\
\hline Environment & Season & Spring & $17.80 \%(212)$ & $<.0001$ \\
\hline variables & & Summer & $39.55 \%(471)$ & \\
\hline & & Autumn & $30.39 \%(362)$ & \\
\hline & & Winter & $12.26 \%(146)$ & \\
\hline & & Unknown & $(2)$ & \\
\hline & Region & South & $46.35 \%(553)$ & $<.0001$ \\
\hline & & Midwest & $26.66 \%(318)$ & \\
\hline & & West & $16.85 \%(201)$ & \\
\hline & & Northeast & $10.14 \%(121)$ & \\
\hline & Location type & Street & $58.26 \%(684)$ & $<.0001$ \\
\hline & & Home & $12.78 \%(150)$ & \\
\hline & & Farm & $12.69 \%(149)$ & \\
\hline & & Public & $9.63 \%(113)$ & \\
\hline & & Sports & $5.88 \%(69)$ & \\
\hline & & Industrial & $0.77 \%(9)$ & \\
\hline & & Unknown & $(19)$ & \\
\hline & Number of users on ATV & 1 & $79.69 \%(938)$ & $<.0001$ \\
\hline & & 2 & $18.27 \%(215)$ & \\
\hline & & 3 & $1.44 \%(17)$ & \\
\hline & & 4 & $0.51 \%(6)$ & \\
\hline & & 5 & $0.08 \%(1)$ & \\
\hline & & Unknown & $(16)$ & \\
\hline & Other vehicle involved & No & $85.37 \%(945)$ & $<.0001$ \\
\hline & & Auto & $10.12 \%(112)$ & \\
\hline & & ATV & $3.43 \%(38)$ & \\
\hline & & Other & $1.08 \%(12)$ & \\
\hline & & Unknown & $(86)$ & \\
\hline
\end{tabular}

* For all $p$ values, we used the chi-square test for equal proportions.

use was location type. Users at home, on the street or farm were significantly less likely to wear helmets than users in other locations (industrial, sports, or public venues) (LogWorth $=11.70, p<0.01$ ). Users operating on the farm, street or at home wore helmets in $7.9 \%$ of cases, compared to users in industrial, sport, or public areas who wore helmets in $32.6 \%$ of cases. Users most likely to have worn helmets were those in public, sports, or industrial locations in the West or Northeast regions of the country, where $50.9 \%$ of users wore helmets compared to only $18.9 \%$ of users in the Midwest or South regions. After four significant partitions, determined by a minimum LogWorth $=2$ $(p=0.01)$, the user demographic least likely to have worn helmets 


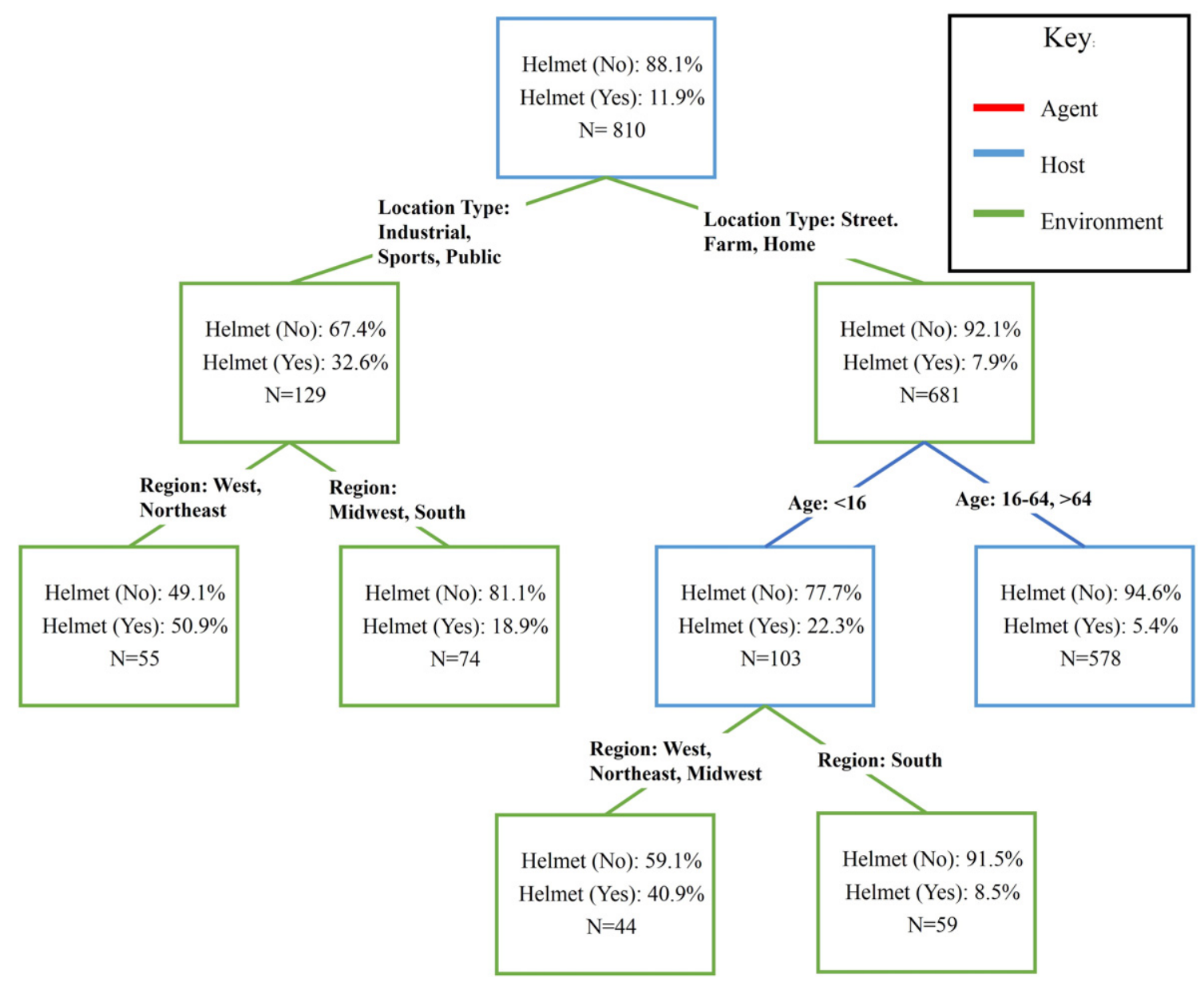

Fig. 1. Helmet use CHAID.

were users operating in the location types of home, street, or farm, who were over the age of 15 . Of these cases, only 31 of 578 (5.4\%) were wearing helmets at the time of mortality, compared to the user demographic most likely to wear helmets, users operating in industrial, sports or public locations in the West or Northeast regions of the country, where 28 of 55 (50.9\%) wore helmets at the time of death.

\subsubsection{Crash type (Fig. 2)}

There were four different categories of ATV crash type: collision, ejection, overturn, and other/unknown. Crashes were significantly more likely to be a collision-type event if another vehicle was involved (LogWorth $=37.22, p<0.01$ ). The percentage of collision-type events rose even further $(87 \%)$ if the crash occurred on the street or in a sports location. Over 150 events categorized as collisions involved trees. The next most common collision was between an ATV and a motor vehicle. This occurred in just over 100 collisions. The third most common collision type was between two ATVs. Other sources of collisions included, fences, poles, deer, and embankments.

When another vehicle was not involved in the loss of control event, location type was a significant covariate associated with crash type (LogWorth $=20.51, p<0.01$ ). When a death occurred on the street, there was a higher percentage of collision and ejection events $(47.4 \%$ and $14.5 \%$ of cases, respectively) and a lower proportion of overturns (30.6\% of cases). Fatal loss of control incidents at locations other than the street (home, farm, sport, industrial, and public) had a higher proportion of overturns (56.1\% of cases), and a lower percentage of collisions and ejections (21.7\% and $12.6 \%$ of cases).

ATV passenger fatalities occurred more often during ejection-type events. As seen in Fig. 2, when another vehicle was not involved in an event occurring on the street, the percentage of fatal ejections for a passenger was $31.4 \%$, while the percentage of fatal ejection for drivers was $12.7 \%$. A further significant split of this branch reveals that drivers over the age of 64 years were more likely to be involved in overturn events (54.8\%), in comparison to those under the age of 65 years $(29.3 \%)$ $($ LogWorth $=3.59, p<0.01)$.

\subsubsection{Location (Fig. 3)}

The covariate most associated with location of fatalities was the type of crash. Users who were involved in a collision or an ejection were more likely to be operating on the street (72.1\%) than users involved in an overturn or other-type event (41.1\%) ( $\log$ Worth $=19.12$, $p<0.01$ ). Operators under 16 or over 64 years comprised the largest percentage of fatalities in overturns or other-type events taking place at homes and on farms. For these age groups, 65\% of fatalities occurred on farms or at home, compared to the overall average of $25.5 \%$ (LogWorth $=2.67, p<0.01$ ).

\section{Discussion}

While our findings support previous research on risk factors for ATV injuries and fatalities, what makes this study unique is the potential for practical applications based on associations between risk factors. Demographic risk factors that have been identified in previous studies include being male (Breslau et al., 2009; Goldcamp et al., 2006; Helmkamp, 2012; Helmkamp et al., 2009; O'Connor et al., 2009; Rechnitzer et al., 2013; Rodgers, 2008; Rodgers \& Adler, 2001), young or old ages (Balthrop et al., 2009; Helmkamp, 2012; Helmkamp \& Carter, 2009; Helmkamp et al., 2011; O'Connor et al., 2009; Rodgers, 2008; Rodgers 


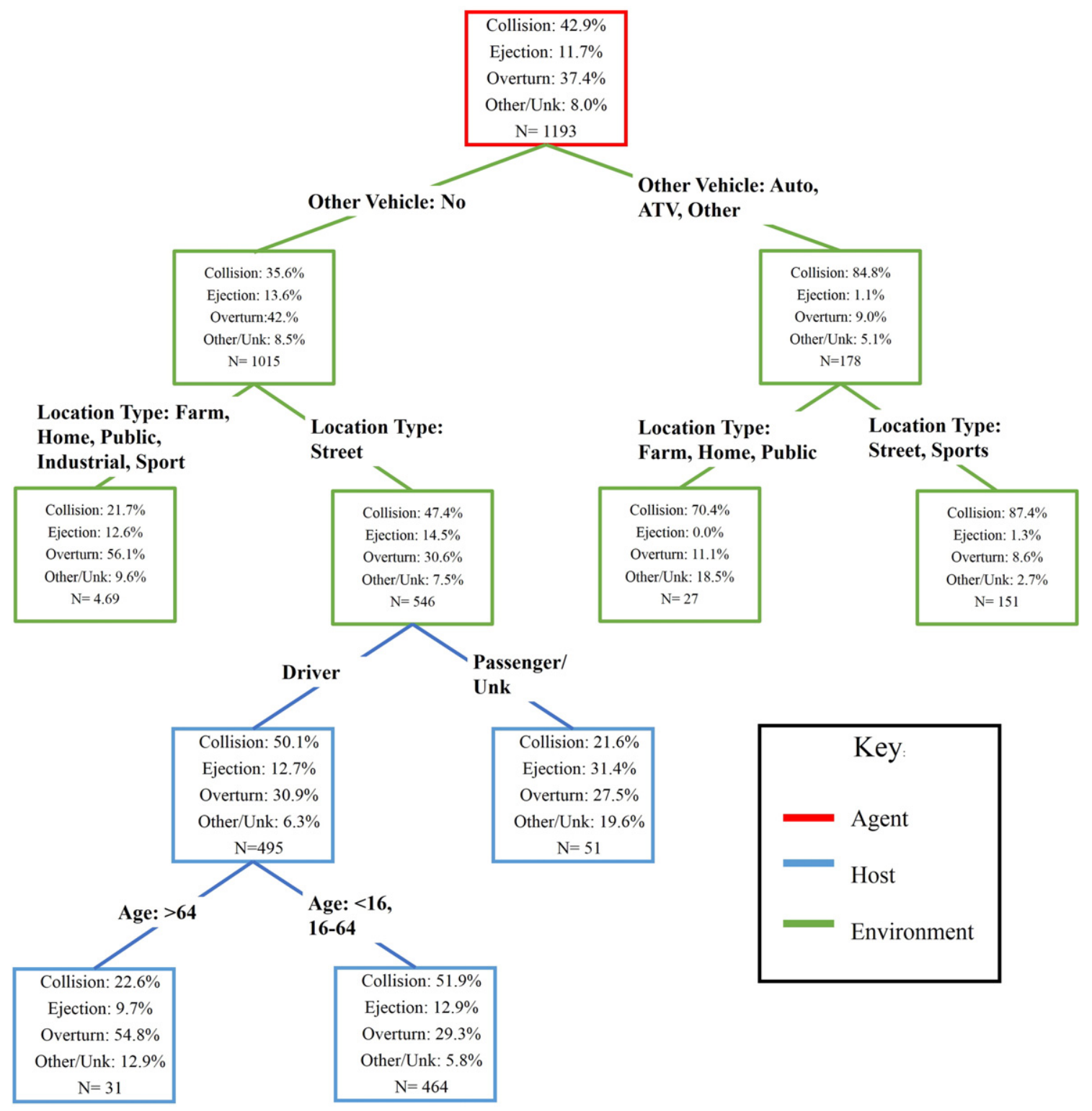

Fig. 2. Crash type CHAID.

\& Adler, 2001), inexperience (Goldcamp et al., 2006; O'Connor et al., 2009; Rodgers \& Adler, 2001), and use of unsafe practices including lack of personal protective equipment (PPE) and using drugs or alcohol (Fleming, 2010).

The high ratio of male to female deaths is not unique to the data contained in this report. Helmkamp (2012) used data from 2000 to 2007 and found $86 \%$ of ATV deaths were among males (Helmkamp, Aitken, Graham, \& Campbell, 2012), a number that is consistent with the results in this study where $85 \%$ of deaths were among males. While a majority of ATV riders are male (Levenson, 2003), males are also more likely to die or be injured in a crash (Levenson, 2003). Helmkamp, Aitken, and Lawrence explained gender differences in both ATV incidents, as well as cycling incidents, as a combination of both exposure and behavior. They concluded that males were more likely to engage in risky behaviors, which contributed to the higher injury rates among males (Helmkamp et al., 2009).

The ATV was designed as an off-road vehicle, with its' low pressure, balloon tires, and suspension system designed to grip uneven survaces (Ford \& Mazis, 1996). However, almost 60\% of fatalities during this period occurred on the street. Additionally, the majority of ATV's are designed for a single rider, yet almost $9 \%$ of fatalities occurred to the passenger of the ATV. Furthermore, there were several fatalities involving an ATV carrying three or more riders.

Another variable related to the host aspect of the epidemiologic triangle, helmet use, has been extensively analyzed in previous studies. Sibley and Tallon (2002) reported helmet use prevalence in a study of ATV injuries in Nova Scotia similar to that in the present study. Sibley and Tallon (2002) also indicated that among riders with severe ATVrelated injuries, helmet use prevalence was 16\%; drug and alcohol use prevalence was 56\% (Sibley \& Tallon, 2002). While our findings are consistent with the reported prevalence of helmet use, drug and alcohol use in ATV injuries and fatalities varies across studies.

Percy and Duffey (1989), who interviewed patients who were admitted to a regional emergency department about ATV injuries, found approximately $30 \%$ of participants reported alcohol use before or during ATV operation (Percy \& Duffey, 1989). This number is significantly different than the results presented in this study, where alcohol and drug use was positive in $84 \%$ of fatalities with a known alcohol and drug status. Possible explanations to this wide range of findings in regard to drug and alcohol use prevalence may be due to the different outcomes (injury vs. fatality) as well as the high number of cases of unknown drug and alcohol involvement in this study. 


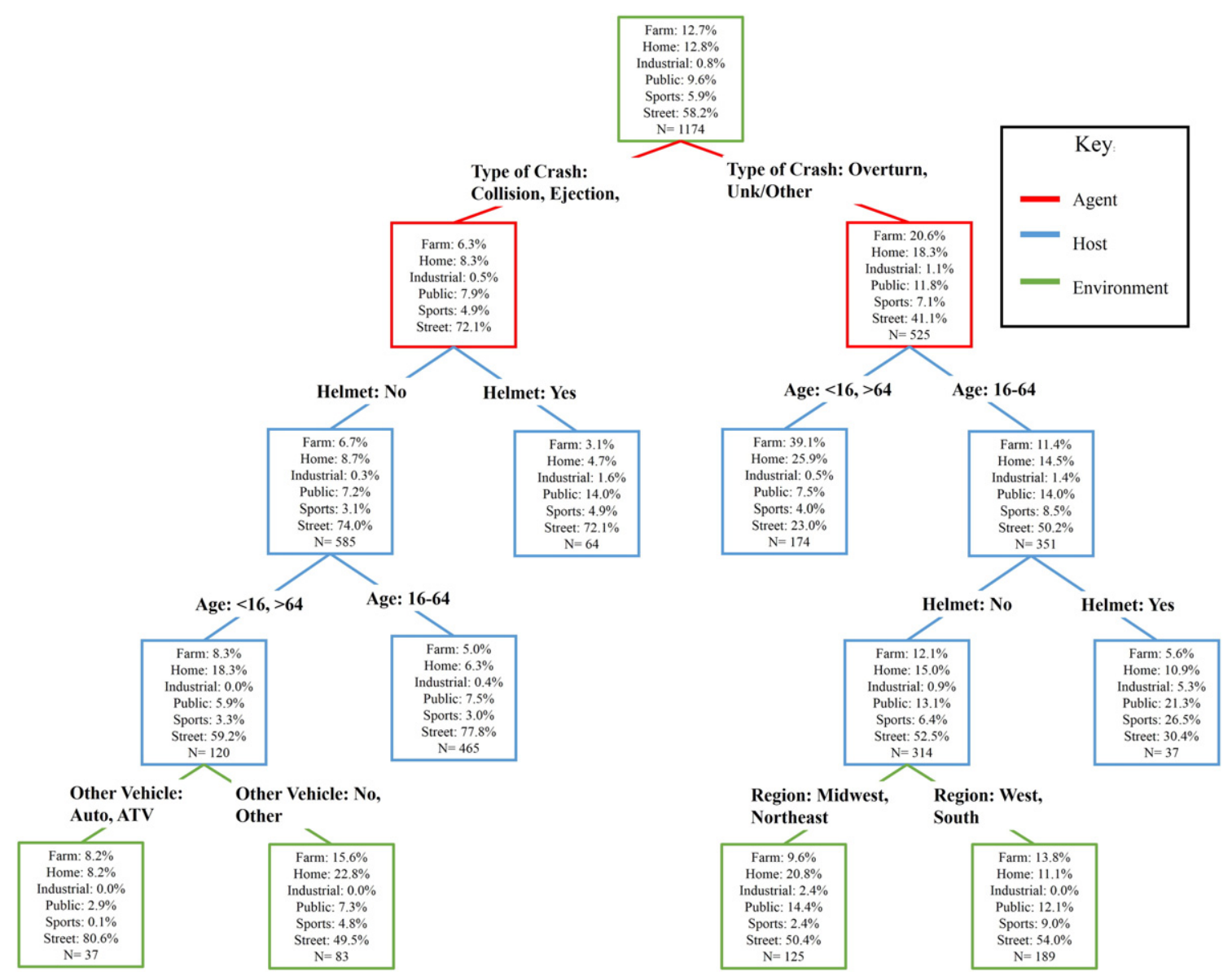

Fig. 3. Location CHAID.

There were many similarities between the results of previous studies and the results of this study for agent and injury-related variables. Garland (2014) found that musculoskeletal and traumatic head injuries were frequent types of injuries resulting from the most common ATV crash types: overturn/rollover and collisions (Garland, 2014). The results of this study corroborate these two mechanisms as the most common. Collisions and overturns were involved in $43 \%$ and $37 \%$ of crashes, respectively. Findings related to the body part injured vary by study and by outcome severity. According to 2010 CPSC data, approximately $38 \%$ of emergency department-treated ATV injuries were a fracture, dislocation, or sprain/strain (Garland, 2014); additionally, brain trauma accounted for $22 \%$ of ATV injuries (Balthrop et al., 2009). The results of this study presented a different distribution of injuries by body part, with the head as the most common body part injured $(60 \%$ of cases). However, as this study analyzed deaths exclusively, a higher proportion of injuries to the head should be expected in comparison to morbidity cases.

As mentioned previously, environmental conditions are less commonly recorded and analyzed in comparison to host and agent factors. The results presented in this study indicated that the location of the incident was an important factor in many of the models. Garland (2014) studied the differences in location on outcome severity and found emergency department-treated injuries were more likely to occur in a field $(20 \%)$ or in the woods $(20 \%)$, while fatalities were more likely to occur on paved (34\%) or unpaved (20\%) roads (Garland, 2014). This information, as well as the results contained in this study, indicate a need for additional research on the role that environmental factors have on the risk of injury and fatality.

We employed a novel application of existing methods to analyze a secondary data set to better understand the interaction between risk factors in ATV deaths. We identified interactions between all three aspects of the epidemiologic triangle related to ATV mortality. Specifically, $44 \%$ of significant partitions were classified host factors and $50 \%$ were classified as environmental factors. Reoccurring partitions within the three CHAID models included significant differences based upon operator's age, location type, and region of operation. These reoccurring partitions point to the need for specific intervention strategies, which account for the different operating behaviors seen in across age groups, operating locations, and regions of the country.

Within the CHAID trees, there are factors that reoccur either within different branches of the same tree or across the different trees. There were two main reoccurring host partitions: age and helmet use. There was only a single instance of an agent variable, crash type, having a significant partition on the location type tree. However, the partition occurred at the top of the CHAID for location type, indicating the highest LogWorth value. The same three environmental variables were responsible for multiple partitions across the three trees. Other vehicle involved, region, and location type, were all responsible for significant partitions in two out of the three trees.

Within the CHAID tree of helmet use, users who operate on the farm, home, or street had lower helmet use than other location types (Fig. 1). Previous research indicates that users who operate on the farm or home may be less likely to wear helmets due to their use of ATVs for occupational purposes (O'Connor et al., 2009). Occupational users may have the perception that helmets are cumbersome and make performing occupational activities difficult (Fragar, Pollock, \& Morton, 2006).

There was a significant difference in the prevalence of helmet use between users under the age of 16 versus users 16 and older who operate on the street, farm, or at home (Fig. 1). This could be attributed to some states enacting legislation that requires helmets for users under 
the age of 16 , or perhaps as a result of provisions of the 2008 CPSIA. As of February 2012, 30 of the 50 states had legislation specific to helmet protection for ATV users (Specialty Vehicle Institute of America, 2012). However, the most common legislation required helmets only to operators under the age of 18 , operating on public lands. While this legislation is a step toward safer ATV use, there is no information on enforcement or compliance with legislation at the state or national level (Specialty Vehicle Institute of America, 2012).

The most significant factor associated with the outcome measure of crash type (Fig. 2) was another vehicle's involvement in the incident. A higher prevalence of collisions in street and in sports locations may be due to increased vehicle traffic at these locations. The higher prevalence of overturns on farms and at home may be due to using the ATV to carry heavy loads to accomplish tasks or due to the installation of aftermarket alterations to the body of the ATV, making the vehicle more prone to rollover (Occupational Safety and Health Administration, 2006).

The only host-related factor significantly associated with type of crash was age. Users over 64 years of age had a higher prevalence of overturns than the other age groups. Correct operation of an ATV involves leaning and shifting body weight and forceful exertion to control the vehicle (Helmkamp, 1999). Poor vision and reduction of muscle strength and coordination may lead to differences in crash dynamics in older users compared to younger users (Helmkamp, 1999).

Location at time of death was significantly associated in all three of the CHAID classification trees. Partitioning within the CHIAD trees often occurred between operation on a farm versus street locations. Occupational use has been associated with different hazards such as use rate (Rodgers \& Adler, 2001), different ATV design (including aftermarket modifications to the vehicle; Occupational Safety and Health Administration, 2006), operation during adverse weather conditions, and the presence of stressors with regard to productivity (Carman et al., 2010). Use of ATVs is likely to be a more common occurrence (higher use hours/year) on farms than users who are operating for recreational purposes (Rodgers \& Adler, 2001).

The reoccurring themes of helmet use and age have been addressed through previous intervention strategies such as the CPSIA, which placed an emphasis on marketing campaigns for helmet use as well as provisions for child and youth riders (Catenacci, 2009; Fleming, 2010; Goldcamp et al., 2006). However, there have been few efforts to assess risk differences in different locations and in different regions of the country. It is imperative to understand the unique training and education needs of different populations in order for intervention strategies to be effective.

\subsection{Limitations}

A primary challenge to modeling the interactions between the many variables associated with ATV fatalities has been access to data and information concerning the incidents. There are two primary methods to obtaining information related to ATV injuries or fatalities, each with major limitations. The first method involves secondary data analysis from hospital databases. Data obtained from this method have limitations in available information regarding crash characteristics, location of crash, helmet use, and generalizability of results to another environmental or geographic area. The second method is based on in-depth investigation (INDP) reports from the Consumer Product Safety Commission. While the second method overcomes limitations related to small sample size and generalizability to the nationwide population, this method is limited to the variables and information collected during the CPSC's investigation. In most cases, CPSC fatality reports contain complete demographic information but limited information related to factors such as the experience of the operator, training, agent-related factors, or environmental conditions.

The CPSC investigations contained in this report primarily originate based off of news reports or death certificate files of incidents. INDP reports target specific variables related to the ATV crash such as helmet use, type of crash, and age. While use of the standardized reporting structure ensures consistent reporting of demographic variables and other targeted variables, many other important data points were missing from the INDP reports. Variables such as alcohol and drug use were reported irregularly and there was little continuity in the information provided in the narrative reports. Factors such as ATV training, experience operating an ATV, and even time of day of the incident, were rarely mentioned in the INDP report, all of which have been determined by prior research to be pertinent factors in probability of loss of control. Bias was suspected in the alcohol use statistics due to a large percentage of reports containing no information on alcohol and drug use. Differential reporting of alcohol/drug was suspected due to the inability to confirm absence alcohol or drug activity and the reports' lack of recording pertinent negative findings.

In addition, due to a lack of nationwide licensing, registration, or training requirements, accurately determining the number of ATV users in the United States is challenging. Without detailed reports of injuries and statistics related to the prevalence of ATV use, it is impossible to accurately determine the rate of ATV fatalities or calculate risk or rate ratios pertaining to specific risk factors.

\section{Conclusions}

This research provides a model for understanding of the relationship between contributing risk factors to ATV fatalities. Combination of the CHAID analysis method and the epidemiologic triangle allows for visualization of the interaction between the host-agent-environment factors and ATV fatalities. These models could later be used as the basis for specific intervention strategies based upon the correlations between risk factors.

Information and analysis beyond demographic variables is needed to advance ATV research and for the continued improvement of safety campaigns and training programs. For example, inexperience is cited as a major risk factor for ATV injury (Goldcamp et al., 2006; O'Connor et al., 2009; Rodgers \& Adler, 2001); however, years of operating experience or training is not part of the standardized CPSC investigation report, making it difficult to understand the influence of these variables on the risk of injury or fatality.

The present study is an example of the interaction that exists between the host, agent, and environment. No one factor is ultimately responsible for the number of fatal cases seen in this report. These interacting elements each build upon each other, some having more impact than others. Ultimately, categorization and association provides a more thorough understanding of the variables contributing to ATV fatalities.

\section{Practical applications}

This report is a representation of the current risks ATVs pose to the user on a national scale. This report should serve as a method of identifying at-risk groups of users to create specialized marketing and training solutions. By modeling associated risk factors and categorizing them as pertaining to the host, agent, or environment, it is possible to develop targeted solutions to the root cause of the hazard. Targeted solutions should account for the specific risks and behaviors associated these distinct groups of users. The results of this paper have identified significant partitions in use behaviors and fatalities based on regional location, demographic characteristics, and location type.

Through use of legislation and training, many host-related risk factors can be addressed. For example, focusing campaigns, legislation, and resources on areas with the greatest need may have a large impact on the helmet use prevalence. For example, resources and campaigns could focus on the youth population in the South where nearly $92 \%$ of youth under the age of 16 were not wearing helmets at the time of the ATV loss of control event. 
Use of engineering controls can mitigate risk due to the agent and/or physical environment. Two examples of engineering controls include: use of speed governors to control the speed capabilities of the ATV, and the transition from three-wheeled ATVs to inherently more stable four-wheel models.

Use of targeted marketing strategies and education may be able to limit risk due to the social environment. For instance, reminders about the designated purpose of the ATV as an exclusively off-road, rather than highway or street vehicle, as well as manufacturer recommendations to limit the number of riders on an ATV to its designed capacity.

There are many other opportunities and possible intervention strategies that may be applied to this public health problem. Use of the Haddon Matrix as a method of developing countermeasures to limit risk during the different phases of social concern has shown to be an effective method of inspiring possible intervention strategies to combat issues of injury epidemiology. For example Haddon's' first phase, the "prevention of mechanical forces above injury thresholds" can be achieved through the use of the engineering control method mentioned previously: speed governors. Haddon's second phase, "interaction of mechanical forces on the host" can be achieved through use of personal protective equipment, such as helmets, to protect riders from experiencing head trauma during a loss of control event. The final phase of social concern, maximizing salvage, has yet to be discussed in context of this project. Perhaps the best way to ensure the survivability of an ATV rider involved in an incident is to ensure the injured ATV user is found and receives prompt professional evaluation and aggressive treatment. Provider education and awareness of the mechanism of injury present during an ATV loss of control event is crucial to appropriate evaluation and treatment.

The present study is an example of the interaction that exists between the host, agent, and environment. No one factor is ultimately responsible for the number of fatal cases seen in this report. These interacting elements each build upon each other, some having more impact than others. Ultimately, categorization and association provides a more thorough understanding of the contributing variables associated with ATV fatalities as well as the opportunity for multiple mitigation strategies during the different phases of the loss of control event.

\section{Funding}

Contract grant sponsor: Centers for Disease Control (CDC)/NIOSH Mountain and Plains Education and Research Center; contract grant number: T42OH009229-04.

Contract grant sponsor: Centers for Disease Control (CDC)/NIOSH High Plains Intermountain Center for Agriculture Occupational Health and Safety Cooperative Agreement No. 2U54 OH008085.

\section{References}

Balthrop, P. M., Nyland, J., \& Roberts, C. S. (2009). Risk factors and musculoskeletal injuries associated with all-terrain vehicle accidents. The Journal of Emergency Medicine, 121-131.

Breslau, J., Stranges, E., Gladden, M., \& Wong, H. (2009). Emergency department visits and inpatient hospital stays for all-terrain-vehicle-related injuries. Healthcare cost and Utilization Project, 130, 1-10.

Carman, A. B., Gillespie, S., Jones, K., Mackay, J., Wallis, G., \& Milosavljevic, S. (2010). All terrain vehicle loss of control events in agriculture: Contribution of pitch, roll and velocity. Ergonomics, 18-29.

Catenacci, M. H. (2009). Changes in the epidemiology of all-terrain vehicle accidents. Southern Medical Journal, 449.

Fleming, S. (2010). All-terrain vehicles: How they are used, crashes, and sales of adult-sized vehicles for children's use. United States Government Accountability Office.

Ford, G. T., \& Mazis, M. B. (1996). Informing buyers of risks: Analysis of the marketing and regulation of all terrain vehicles. Journal of Consumer Affairs, 30(1), 90-123.

Fragar, L. J., Pollock, K., \& Morton, C. (2006). ATV injury on Australian farms: The facts. Facts and figures on farm health and safety series no 8.

Garland, S. (2014). National estimates of ATV-related emergency department-treated injuries. United States Consumer Product Safety Commission Injury Statistics.

Goldcamp, E. M., Myers, J., Hendricks, K., Layne, L., \& Helmkamp, J. (2006). Nonfatal allterrain vehicle-related injuries to youths living on farms in the United States, 2001. National Rural Health Association, 22(4), 308-313.
Haddon, W. (1968). The changing approach to the epidemiology, prevention, and amelioration of trauma: The transition to approaches etiologically rather than descriptively based. American Journal of Public Health and the Nation's Health, 58(8), 1431-1438.

Helmkamp, J. (1999). All-terrain vehicle-related deaths among the West Virginia elderly, 1985 to 1998. American Journal of Public Health, 89(8), 1263-1264.

Helmkamp, J. (2012). All-terrain vehicles and work. NIOSH Science Blog.

Helmkamp, J., \& Carter, M. W. (2009). ATV deaths among older adults in West Virginia: Evidence suggesting that "60 is the new 40!". Southern Medical Journal, 120(5), 465-469.

Helmkamp, J., Aitken, M. E., Graham, J., \& Campbell, C. R. (2012). State-specific ATVrelated fatality rates: An update in the new millennium. Public Health Reports (1974), 127(4), 364-374.

Helmkamp, J., Aitken, M. E., \& Lawrence, B. A. (2009). ATV and bicycle deaths and associated costs in the United States, 2000-2005. Public Health Reports, 124, 409-418.

Helmkamp, J., Marsh, S. M., \& Aitken, M. E. (2011). Occupational all-terrain vehicle deaths among workers 18 years and older in the United States, 1992-2007. Journal of Agricultural Safety and Health, 147-155.

Hulme, A., \& Finch, C. F. (2015). From monocausality to systems thinking: A complementary and alternative conceptual approach for better understanding the development and prevention of sports injury. Injury Epidemiology, 2(1), 1-12. http://dx.doi.org/10. 1186/s40621-015-0064-1.

Landis, J. R., \& Koch, G. G. (1977). The measurement of observer agreement for categorical data. Biometrics, 159-174.

Levenson, M. S. (2003). All-terrain vehicle 2001 injury exposure studies. Retrieved from https://www.cpsc.gov/PageFiles/102410/atvex2001.pdf.

Mangus, R. S., Simons, C. J., Jacobson, L. E., Streib, E. W., \& Gomez, G. A. (2004). Current helmet and protective equipment usage among previously injured ATV and motorcycle riders. Injury Prevention: Journal of the International Society for Child and Adolescent Injury Prevention, 10(1), 56-58. http://dx.doi.org/10.1136/ip.2003.002626.

Myers, M. L., Cole, H. P., \& Mazur, J. M. (2009). Cost effectiveness of wearing head protection on all-terrain vehicles. Journal of Agromedicine, 14(3), 312-323. http://dx.doi.org/ 10.1080/10599240903041885.

Occupational Safety and Health Administration (2006). Hazards associated with allterrain vehicles (ATVs) in the workplace. Retrieved from https://www.osha.gov/ dts/shib/shib080306.html.

O'Connor, T., Hanks, H., \& Steinhardt, D. (2009). All-terrain vehicle crashes and associated injures in North Queensland: Findings from the rural and remote road safety study. The Australian Journal of Rural Health, 251-256.

Percy, E. C., \& Duffey, J. P. (1989). All-terrain-vehicle injuries. A sport out of control Clinical Medicine, 296-299.

Rechnitzer, G., Grzebieta, R. H., McIntosh, A. S., \& Simmons, K. (2013). Reducing all terrain vehicle injuries (ATVs) and deaths- a way ahead. Paper presented at the International Technical Conference the Enhanced Safety of Vehicles (ESV).

Rodgers, G. B. (2008). Factors associated with the all-terrain vehicle mortality rate in the United States: An analysis of state-level data. Accident Analysis and Prevention, 40, $725-732$.

Rodgers, G. B., \& Adler, P. (2001). Risk factors for all-terrain vehicle injuries: A national case-control study. American Journal of Epidemiology, 153(11), 1112-1118.

Runyan, C. W. (2003). Introduction: Back to the future-revisiting Haddon's conceptualization of injury epidemiology and prevention. Epidemiologic Reviews, 25(1), 60-64. http://dx.doi.org/10.1093/epirev/mxg005.

SAS Institute Inc. (2014a). Specialized models: Partition method: Statistical details. Retrieved from http://www.jmp.com/support/help/Statistical_Details_9.shtml\#1198372

SAS Institute Inc. (2014b). Usage note 24329-In the SAS Enterprise Miner Tree node, what is the logworth statistic? Retrieved from http://support.sas.com $/ \mathrm{kb} / 24 / 329 . \mathrm{html}$.

Sibley, A. K., \& Tallon, J. M. (2002). Major injury associated with all-terrain vehicle use in Nova Scotia: A 5-year review. Canadian Journal of Emergency Medical Care, 4(4), 263-267.

Specialty Vehicle Institute of America (2012). State all-terrain vehicle requirements. SPSS Inc. (2002). Introduction to AnswerTree. Chicago, IL

Topping, J., \& Garland, S. (2014). 2012 Annual report of ATV-related deaths and injuries. US Consumer Product Safety Commission.

Elise Lagerstrom, MS is a Doctoral Student and Graduate Research Assistant at Colorado State University in the Environmental Health program with a specialization in ergonomics. Elise Lagerstrom has a BS in Human Factors Psychology from Embry-Riddle Aeronautical University and a MS in Environmental Health from Colorado State University. The focus of her work involves ATV safety and the analysis of risk factors and environmental conditions leading to injuries and fatalities.

Sheryl Magzamen, MPH, PhD. Dr. Magzamen's primary research focus is understanding the relative contribution of social factors and environmental exposures on childhood chronic disease. She has worked extensively in the elementary school setting on developing surveillance methods for childhood asthma, understanding the role of lead exposure in educational outcomes, and analyzing the role of indoor environmental quality and student health and performance. She has active collaborations with exposure scientists to develop refined exposure assessment models in community and agricultural settings in studies of respiratory disease. Her current methodological work focuses on the application of novel approaches to understand environmental pollutant mixtures in community-based studies.

Lorann Stallones, MPH, PhD is a Professor in the Department of Psychology, Colorado State University (CSU), Director of the Colorado Injury Control Research Center, and Director of the Colorado School of Public Health at CSU, part of the collaborative Colorado Schoo of Public Health. She was selected as a Professor Laureate in the College of Natural Sciences in 2011. She received the Faculty Excellence in Research Award from the Colorado School 
of Public Health in 2011.

David Gilkey, DC PhD. Dr. Gilkey is an Associate Professor of Environmental and Occupational Heath at Colorado State University with expertise in occupational health, workplace wellness, ergonomics, and safety. He earned his Doctor of Chiropractic degree from Southern California Health Sciences University and PhD from CSU with a focus in occupational health, safety, industrial hygiene and ergonomics. He is a Certified Professional Ergonomist (CPE) and Certified Safety Specialist (CSP). His research has focused in construction safety and agriculture. He has also been involved in translational research (R2P) looking at methods to evaluate and enhance safe work practices in agriculture.
John Rosecrance, PT, PhD. Dr. Rosecrance is Professor of Safety and Ergonomics at Colorado State University and at the Colorado School of Public Health. He has graduate degrees in occupational biomechanics from the University of North Carolina and in occupational ergonomics from the University of Iowa. Dr. Rosecrance is a Certified Professional Ergonomist and Physical Therapist involved in teaching and consulting on the prevention of musculoskeletal injuries. His research involves work processes in the manufacturing, construction, and agricultural sectors. His current research topics include safety leadership, safety and ergonomics culture, ergonomic exposure assessment, and efficient work methods. 